$\begin{array}{ll}\text { Italique } & \text { Italique } \\ \text { Poésie italienne de la Renaissance }\end{array}$

VI| 2003

Varia

\title{
Piccolomini, Braccesi e Achillini : dal latino al volgare, dalla prosa al verso
}

\section{Andrea Comboni}

\section{(2) OpenEdition}

\section{Journals}

\section{Edizione digitale}

URL: http://journals.openedition.org/italique/139

DOI: 10.4000/italique.139

ISSN: 1663-4438

\section{Editore}

Librairie Droz

\section{Edizione cartacea}

Data di pubblicazione: 31 dicembre 2003

Paginazione: $37-51$

ISBN: 2-600-00893-4

ISSN: 1423-3983

\section{Notizia bibliografica digitale}

Andrea Comboni, « Piccolomini, Braccesi e Achillini : dal latino al volgare, dalla prosa al verso », Italique [Online], VI | 2003, online dal 06 octobre 2009, consultato il 22 avril 2019. URL : http://

journals.openedition.org/italique/139; DOI : 10.4000/italique.139 


\section{A N D REA COMBONI}

P I C C O L O M I N I, B R A C C E S I E A C H I L L I N I:

D A L L A T I O A L V O L G A R, D A L L A ROSA A L VERO 


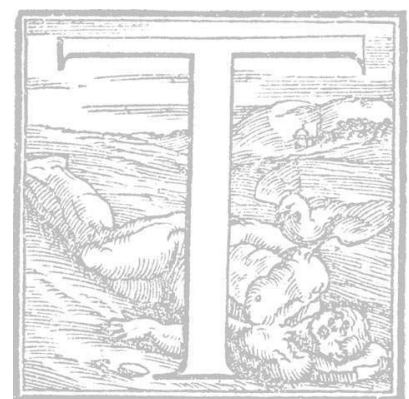

$R a$ le rime del bolognese Giovanni Filoteo Achillini (I466-I538), solo in anni recenti, grazie a un fortunato recupero, sottratte all'oblio, ${ }^{\mathrm{I}}$ si leggono sonetti, sestine, capitoli ternari, barzellette. Degli ottantotto componimenti contenuti nel ms. Acquisti e Doni 397 della Biblioteca Medicea Laurenziana di Firenze, ben trentadue risultano capitoli in terza rima. La maggioranza di questi ternari (in numero di venti) è riconducibile al genere elegiaco. E noto, infatti, come il capitolo ternario, dopo le 'anteprime' offerte da Leon Battista Alberti e Giusto de' Conti, divenga tra Quattro e Cinquecento il metro più frequentemente impiegato per la composizione di elegie in volgare. ${ }^{2} L$ ' Achillini elegiaco mostra, in particolare, una chiara preferenza per il sottogenere, allora in gran voga, dell'epistola amorosa, al quale appartengono ben dodici dei suoi ternari. ${ }^{3}$ Se il modello primo a cui fanno riferimento le epistole amorose del letterato bolognese è generalmente costituito, com'è del tutto ovvio, dalle Heroides ovidiane, ${ }^{4}$ nella composizione dell'epistola Prestantissima sopra ogni

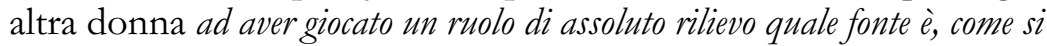
vedrà, un testo quattrocentesco in prosa. Ma si legga, innanzitutto, il ternario in questione:

Prestantissima sopra ogni altra donna

In virtute, beltate e ligiadria,

E d'ogni humanità firma colonna,

Scrivendo in questa te salutaria,

Se di salute havesse parte alcuna

Ma da te pende ogni salute mia.

Io te amo più di me poiché Fortuna

Questo dispone, anci è il fiero Amore

Che ogni sua forza contra me raduna.

Per fede e segurtà ti dedi il core,

Li testimonii son pianti e suspiri,

Il ver contracto è solo il mio dolore.

Supplico dunque che tu non te adiri

Poiché mia fede me ti fa constante,

Ançi pietosa facte a mei martyri.

Acceptame per servo e fido amante,

Contentate ch'io t'ami, o chiara stella,

E fie restauro alle mie pene tante.

La gentilezza tua, vaga angelella,

M'ha vinto al tutto e factome pregione

A tua persona sopra ogni altra bella. 
Di me l'arbitrio et ogni mia ragione

T'ho dato in preda e il corpo e l'alma e il senso,

E sol di questo tua beltà è cagione.

E giorno e nocte, e nocte e giorno io penso

In te sola, e te sola invoco e chiamo,

Il cor ti dedi per tributo e censo.

Desidero te sola e te sola amo,

In te consiste tutto il mio dilecto:

A te me dono e sol d'esser tuo bramo.

Vivo in te sola, e nel tuo vivo pecto

Vive contento il cor mio catenato

Che ti fa fede ch'io ti sum sugiecto.

Tu sola me pòi bene haver salvato

E darmi morte: ellegi quel che vòi,

Che non serò da te mai discordato.

Deh, famme digno de resposta, e poi

Se Morte virà ben, serò contento,

Ma prego che me aiuti perché pòi.

Cum la resposta allenta il mio tormento,

Facte benigna come mostri in volto,

Perché 'l benigno ogni gran male ha spento.

In questo mio parlar non chiedo molto

(E a me fie molto): solo io te domando

Narrarti il foco che ho nel cor sepolto.'

Si tratta di un testo in cui a parlare è una voce maschile, così come si verifica altre sette volte nell'epistole dell'Achillini. Nel caso in questione lo scrivente ricorre allo strumento dell'epistola per pronunciare una solenne dichiarazione d'amore alla donna che ha conquistato il suo cuore. Il componimento a una prima lettura può risultare un concentrato di affermazioni e situazioni topiche: una delle tante (per non dir troppe) declinazioni quattro-cinquecentesche del codice elegiaco. In realtà, grazie a una fortunata "agnizione di lettura", 6 è possibile riconoscere in quest'epistola dell'Achillini una sorta di versione volgarizzata in terza rima di una delle lettere contenute nell'Historia de duobus amantibus di Enea Silvio Piccolomini (destinato a salire al soglio pontificale con il nome di Pio II), opera di intonazione elegiaca e autentico best-seller del tardo Quattrocento. ${ }^{7}$ Le epistole presenti nell'Historia risultano, come è stato giustamente osservato, «veri e propri brani di un ideale formulario di epistolografia galante da offrire al pubblico dei lettori». ${ }^{8}$ Il ternario Prestantissima sopra ogni altra donna risulta fedelmente modellato sulla prima epistola inviata da Eurialo a Lucrezia, per dichiararle tutto il suo amore: 
Salutarem te, Lucretia, meis scriptis, si qua mihi salutis copia foret. Sed omnis tum salus tum vite spes mee ex te pendet. Ego te magis quam me amo nec te puto latere meum ardorem. Lesi pectoris iudex tibi esse potuit vultus meus, sepe lacrimis madidus et que te vidente emisi suspiria. Fer benigne, te precor, qui me tibi aperio. Cepit me decus tuum vinctumque tenet eximia, qua omnibus prestas venustatis gratia. Quid esset amor, antehac nescivi, tu me cupidinis imperio subiecisti. Pugnavi diu, fateor, violentum ut effugerem dominium. Sed vicit meos conatus splendor tuus, vicerunt oculorum radii, quibus es sole potentior. Captivus sum tuus nec iam mei amplius compos sum, tu mihi et somni et cibi usum abstulisti. Te dies noctesque amo, te desidero, te voco, te expecto, de te cogito, te spero, de te me oblecto; tuus est animus, tecum sum totus, tu me sola servare potes solaque perdere. Elige horum alterum et quid mentis habeas rescribe. Nec durior erga me verbis esto quam fueras oculis, quibus me colligasti. Non peto rem grandem: ut alloquendi te copiam habeam, postulo. Hoc tantum volunt he littere, ut que nunc scribo dicere possim coram. Hoc si das, vivo et felix vivo. $\mathrm{Si}$ negas, extinguitur cor meum, quod te magis quam me amat. Ego me tibi et tue commendo fidei vale, anime mi et vite subsidium mee. ${ }^{9}$

Si può facilmente constatare come la lettera di Eurialo sia il testo da cui si è generata l'epistola dell' Achillini. Identici risultano, infatti, l'ordine e lo svolgimento degli argomenti (e in alcuni casi, inoltre, $i$ versi del letterato bolognese si rivelano traduzioni letterali del testo di Enea Silvio):

Scrivendo in questa te salutaria, Se di salute havesse parte alcuna Ma da te pende ogni salute mia. Io te amo più di me [...]

$$
\text { (vv. 4-7) }
$$

Per fede e segurtà ti dedi il core, Li testimonii son pianti e suspiri,

(Vv. IO-I I)

Supplico dunque che tu non te adiri Fer benigne, te precor, qui me tibi aperio.

Poiché mia fede me ti fa constante,

Ançi pietosa facte a mei martyri.

$$
\text { (VV. I3-I 5) }
$$

La gentilezza tua, vaga angelella,

M'ha vinto al tutto e factome pregione

A tua persona sopra ogni altra bella.

(VV. I9-2I) Cepit me decus tuum vinctumque tenet eximia,
qua omnibus prestas venustatis gratia.
Salutarem te, Lucretia, meis scriptis, si qua mihi salutis copia foret. Sed omnis tum salus tum vite spes mee ex te pendet. Ego te magis quam me amo

Lesi pectoris iudex tibi esse potuit vultus meus, lacrimis madidus et que te vidente emisi suspiria. 
Di me l'arbitrio et ogni mia ragione Captivus sum tuus nec iam mei amplius compos sum

T'ho dato in preda e il corpo e

l'alma e il senso

$$
\text { (vv. 22-23) }
$$

E giorno e nocte, e nocte e giorno Te dies noctesque amo, te desidero, te voco, te io penso expecto, de te cogito, te spero, de te me oblecto;

In te sola, e te sola invoco e tuus est animus, tecum sum totus chiamo,

Il cor ti dedi per tributo e censo.

Desidero te sola e te sola amo,

In te consiste tutto il mio dilecto:

A te me dono e sol d'esser tuo bramo.

$$
\text { (vv. 25-30) }
$$

Tu sola me pòi bene haver salvato

tu me sola servare potes solaque perdere. Elige

E darmi morte: ellegi quel che horum alterum et quid mentis habeas rescribe. vòi,

Che non serò da te mai discordato.

Deh, famme digno de resposta [...]

$$
\text { (vv. 34-37) }
$$

Cum la resposta allenta il mio tormento,

Facte benigna come mostri in volto

$$
\text { (vv. 40-4I) }
$$

In questo mio parlar non chiedo Non peto rem grandem: ut alloquendi te copiam molto habeam

(E a me fie molto): solo io te postulo. Hoc tantum volunt he littere, ut que nunc domando

Narrarti il foco che ho nel cor scribo dicere possim coram. sepolto

L'Achillini, da buon umanista, non incontrava naturalmente alcuna difficoltà a leggere un testo in latino, ma sul suo scrittoio con ogni probabilità doveva trovarsi non solo o non tanto un esemplare della Historia de duobus amantibus, quanto piuttosto un incunabolo del volgariz:amento-rifacimento di essa ad opera del fiorentino Alessandro Braccesi (I445-I503), ' forse proprio quello stampato a Bologna da Ercole Nani il 3 I agosto I492. Il confronto tra $i$ testi è in grado di fugare, credo, ogni dubbio al riguardo: 


\section{Piccolomini, Braccesi e Achillini}

Prestantissima sopra ogni altra donna

In virtute, beltate e ligiadria,

E d'ogni humanità firma colonna,

Scrivendo in questa te salutaria,

Se di salute havesse parte alcuna

Ma da te pende ogni salute mia.

Io te amo più di me poiché Fortuna

Questo dispone, anci è il fiero Amore

Che ogni sua forza contra me raduna.

Per fede e segurtà ti dedi il core,

Li testimonii son pianti e suspiri,

Il ver contracto è solo il mio dolore. Supplico dunque che tu non te adiri

Poiché mia fede me ti fa constante,

Ançi pietosa facte a mei martyri.

Acceptame per servo e fido amante,

Contentate ch'io t'ami, o chiara stella,

E fie restauro alle mie pene tante.

La gentilezza tua, vaga angelella,

$M$ 'ha vinto al tutto e factome pregione

A tua persona sopra ogni altra bella.

Di me l'arbitrio et ogni mia ragione

T'ho dato in preda e il corpo e l'alma e il senso,

E sol di questo tua beltà è cagione.

E giorno e nocte, e nocte e giorno io penso

In te sola, e te sola ignvoco e chiamo,

Il cor ti dedi per tributo e censo.

Desidero te sola e te sola amo,

In te consiste tutto il mio dilecto:

A te me dono e sol d'esser tuo bramo.

Vivo in te sola, e nel tuo vivo pecto

Vive contento il cor mio catenato

Che ti fa fede ch'io ti sum sugiecto.

Tu sola me pòi bene haver salvato

E darmi morte: ellegi quel che voì,
Lucretia mia, prestantissima et sopra tutte l'altre donne formosissima, io ti saluterei con questa mia epistola se in me fussi parte alcuna di salute, perché ciascuna mia speranza et salute di vivere da te sola dipende. Amo te più che me stesso et credo che 'l mio ardore non ti sia nascoso. El volto mio spesso di lachrime bagnato et e gravissimi sospiri che ho messi in tua presentia

ti possono essere verissimi testimonii della mia

acerba ferita. Priegoti adunque, dapoi che la sorte

m'ha condocto a questo grado, che non babbi a sdegno ricevermi per tuo fidele amante. Consenti, o lucentissima et benignissima stella, che el tuo Eurialo sia tuo amante. La tua immensa belleza $m$ 'ha facto ad te prigione et privato di ciascuna libertà; la serena et angelica faccia tua m'ha legato

con indissolubili cathene. Sono facto servo tuo, più

non sono stato in mio arbitrio, giorno e nocte penso a te. Amo te sola, te desidero, te invoco et chiamo. Di te penso, in te spero, in te consiste ogni mio dilecto. Tuo è l'animo, la vita, lo stato et ogni

mia facultà. Tutto son teco, in te vivo, in te mi Tu sola mi poi salvare et farmi perire:

elegi di queste due cose quella che più ti piace et degnati rispondermi quale sia la voluntà tua. Né mi volere con la risposta essere più dura che mi sia

stata con gli occhi, non ti richiegio di cosa difficile o grande: solo ti domando per gratia che mi conceda poterti alquanto parlare. Questo solamente

vogliono le mie lectere, cioè che io ti possa dire a

bocca quello che al presente ti scrivo. ${ }^{\text {I }}$ 
Che non serò da te mai discordato.

Deh, famme digno de resposta, e poi

Se Morte virà ben, serò contento,

Ma prego che me aiuti perché pòi.

Cum la resposta allenta il mio tormento,

Facte benigna come mostri in volto,

Perché 'l benigno ogni gran male ha spento.

In questo mio parlar non cbiedo molto

(E a me fie molto): solo io te domando

Narrarti il foco che ho nel cor sepolto.

Fin dall'incipit l'Achillini mostra di riferirsi al volgarizzamento-rifacimento del Braccesi: il reimpiego del superlativo prestantissima, di cui non v'è traccia nel testo del Piccolomini, è già un segnale eloquente. Anche la giustificazione della mancanza di salutatio rivela la sua stretta dipendenza dal testo del volgarizzamento: "Scrivendo in questa te salutaria, / Se di salute havesse parte alcuna, / Ma da te pende ogni salute mia», cfr. Braccesi «io ti saluterei con questa mia epistola se in me fussi parte alcuna di salute, perché ciascuna mia speranza et salute di vivere da te sola dipende» (Historia, «Salutarem te, Lucretia, meis scriptis, si qua mibi salutis copia foret. Sed omnis tum salus tum vite spes mee ex te pendet»). L'invito rivolto alla donna del v. I6 «Acceptame per servo e fido amante» trova riscontro solo nel rifacimento del Braccesi («non habbi a sdegno ricevermi per tuo fidele amante»). Il vocativo del v. I7 ("ro chiara stella»), che non ha riscontro nell'Historia, è una riduzione di «o lucentissima et benignissima stella» del testo del Braccesi, cosi come quello del v. In ("vaga angelella») è stato ricavato dal sintagma «angelica faccia» del volgarizzamento. La dittologia verbale del v. 26 «te sola invoco e chiamo» trova riscontro soltanto nel Braccesi ("te invoco et chiamo»), l'intero $v$. 29 «in te consiste tutto il mio dilecto» è un fedele ricalco di «in te consiste ogni mio diletto» (nell'Historia si legge «de te me oblecto»). Il sintagma "Vivo in te» (v. 3I) non trova riscontro nell'Historia ma riprende l' «in te vivo» del volgarizzamento. Anche il «famme digno de resposta» del v. 37 discende da «degnati rispondermi» del Braccesi (Historia, «quid mentis habeas rescribe»). Possiamo quindi concludere che la fonte dell'epistola Prestantissima sopra ogni altra donna è la prima lettera di Eurialo a Lucrezía secondo la versione-rifacimento del Braccesi. E quando l'Achillini decise di inserire questo suo testo all'interno del Viridario, poema in ottave di argomento classico e mitologico, pubblicato a Bologna nel ISI $3,{ }^{\mathrm{I2}}$ lo sottopose a una vera e propria revisione testuale. La nuova redazione del componimento risultò abbreviata (34 versi contro gli almeno 46 della prima stesura) e ritoccata in numerosi punti. Nel Viridario l'epistola in questione figura come scritta da Glauco, figlio di Minosse, all'amata Laura: 
Excelsa donna sopra ogn'altra donna

In leggiadria e virtude a nostra etade,

E d'ogni humanità ferma colonna,

Saluterei tua magna venustade

Se di salute havesse io parte alcuna

Ma posta è mia salute in tua beltade.

Più che me proprio t'amo, ché Fortuna

Questo dispone, anzi è quel fier Cupido

Che ogni sua forza contra me raduna.

Il cor per fede e segurtà t'affido,

Li testimonii son pianti e suspiri,

Il contratto è il dolor che dentro annido.

Supplico dunque che tu non te adiri

Poiché mia fede me ti fa constante,

Anzi piatosa fatte a mei martyri.

Accettame per servo non che amante,

Contentate ch'io t'ami, o chiara stella,

$\mathrm{E}$ fia restauro alle mie pene tante.

La gentilezza tua, vaga angelella,

M'ha vinto al tutto e fattome pregione

A tua bella beltà sopra ogni bella.

Ogni mio arbitrio, forza, ogni ragione

T'ho dato in preda, il corpo, l'alma e 'l senso,

Replicar non accade la cagione.

E giorno e notte, e notte e giorno io penso

In te sola e te sola invoco e chiamo,

Cossì parte del mio gran mal compenso.

Sol d'esser degno de risposta bramo,

Seguane Morte poi ch'io me contento,

$\mathrm{O}$ bona o rea più che la vita l'amo.

Se fia benigna il mio mal serà spento,

Se fia crudel Morte mi fia remedio:

Dunque il responder tuo non mi sia lento.

Altro non dico che 'l dir longo è tedio. ${ }^{13}$

La fondamentale ragione del processo variantistico a cui è stata sottoposta l'epistola consiste nella volontà dell' Achillini di rendere meno evidente, soprattutto nelle prime due terzine, il testo-fonte da cui essa deriva (cfr. i vv. I-2, 4, 6, 8, 16, 24, 28-29). E dalla versione del Braccesi che il letterato bolognese cerca di allontanarsi nella nuova redazione del componimento pubblicato nel Viridario. Data la significativa fortuna che anche al volgarizzamento-rifacimento del fiorentino era toccata in sorte, doveva risultare piuttosto agevole ai lettori del tempo riconoscere nell'epistola dell' Achillini una versione volgarizzata in 
terza rima di un testo in prosa ben noto, dato che, come ho precedentemente ricordato, si trattava della prima lettera in cui ci s'imbatte leggendo l'Historia. Il letterato bolognese, nonostante l'introduzione di una nutrita serie di varianti, non riusci a mimetizara e a occultare il testo-fonte, ma soltanto a renderne un po' meno evidente ed immediato il riconoscimento.

La lettura dell'Historia (e della versione procuratane dal Braccesi) non suggeri all'Achillini soltanto la composizione di Prestantissima sopra ogni altra donna, ma esercitò probabilmente il suo influsso anche nella stesura del capitolo elegiaco Maraviglia non è, mia alma diva. In questo ternario, contenuto anch'esso nel manoscritto laurenziano Acquisti e Doni $397^{\mathrm{I} 4}$ e finora mai pubblicato, si dà spazio a una dettagliata e articolata descriptio pulchritudinis della donna amata. ${ }^{15}$

Maraviglia non è, mia alma diva,

Ch'io m'arrendesse nel tuo primo sguardo,

Ché Amor di forza e d'intellecto priva.

Nel pecto me impiagò con l'aureo dardo,

Né possibil mi fu porme a deffesa,

Ché Amor veloce è più che tigro o pardo.

A contemplar la treccia d'oro accesa

Mi senti' l'alma e il corpo incatenato,

Onde ligato è il corpo e l'alma è presa.

E remirando poi da l'altro lato

La spaciosa fronte signorile

Subito l'alma e il cor ti hebbi donato.

E l'inarcate ciglia, ogniun sutile,

Negre e liggiadre furno a puncto quelle

Che me fèn servo a te, diva gentile.

E gli occhi mansueti, anzi doe stelle

Chiare e lucenti, me obombrorno tanto

Ch'io me contento de le mie fiammelle.

Il nasettino profilato alquanto,

Depinto in quel bel volto fa sì che io

Volontier verso il doloroso pianto.

L'aspecto de le guancie humile e pio,

Che doe rosette assembra in doi bei gigli,

$\mathrm{Fa}$ che 'n te penso e me pono in oblio.

E la beltà di quei labri vermigli

Che dolci debbono esser più che 'l mèle,

Me sforza ch'al mio mal lieto me appigli.

Quel fiato che de Amor gonfia le vele

E avanza il musco ne l'odor suave 
Fàme a me traditor, a te fidele.

Tanto ornamento quella bocchina have

Da quei denti di perle de oriente,

Ch'io legier tengo il mio dolor sì grave.

Le parolette tanto ornatamente

Formate da arte insieme e da Natura

Fan ch'io triompho in questa fiamma ardente.

Il mentin tondo e fesso, in cui fé cura

Il cielo a farlo sì raccolto, è quello

Che fa mia vita in morte, se assicura.

Il collo ben proporzionato e bello,

Ove la bianca gola se riposa,

Me fa constante in questo mio flagello.

Ahimè quel pecto candido, in cui posa

Fra le mammelle picole il mio core,

Ogni hor più incende mia voglia amorosa.

La longa man, che spesso per errore

Per neve è tolta da chi la remira,

Fa sì ch'io laudo del mio stratio Amore.

Quella gentil persona che a sé tira

Ogni costume dal superno choro

Fa che de Amor me fo bersaglio e mira.

Taccio le parti ascose, per cui moro,

Ché dire il tutto sempre mai non lice.

Me intenda sol chi sente il dardo d'oro.

Ma tu, mia dea, mia stella e mia fenice,

Poi che constante il sono e fidel servo,

Prego me facci hormai lieto e felice.

Come il chiar fonte il sitibondo cervo,

Non manco per virtù che per beltade

Fervente assai te bramo, cerco e observo.

In cor gentil non sta ben crudeltade,

Ché crudeltà la bona fama offende,

Sì che, mia diva, mòvate a pietade

La fe' d'un che fidelmente se arrende.

In questa descrizione della bellezza della donna amata l'Achillini applica il canone lungo, al quale anche il Piccolomini si era attenuto per rappresentare all'inizio dell'Historia l'aspetto fisico della figura di Lucrezia:

Statura mulieris eminentior reliquis, come illi copiose et aurei laminis similes, quas non more virginum retrofusas miserat, sed auro gemmisque incluserat. Frons alta spatiique decentis, nulla intersecta ruga, supercilia in arcum tensa, pilis 
paucis nigrisque, debito intervallo disiuncta. Oculi tanto splendore nitentes, ut in solis modum respicientium intuitus hebetarent. His illa et occidere quos voluit poterat et mortuos cum libuisset in vitam resumere. Nasus in filum directus, roseas genas equali mensura discriminabat. Nihil his genis amabilius, nihilque delectabilius visu, que cum mulier risit, in parvam utrimque dehiscebant foveam. Nemo has vidit, qui non cuperet osculari. Os parvum decensque, labia corallini coloris ad morsum aptissima, dentes parvuli et in ordinem positi ex cristallo videbantur, per quos tremula lingua discurrens non sermonem sed armoniam suavissimam movebat. Quid dicam mentis speciem aut gule candorem? Nihil illo in corpore non laudabile. Interioris forme indicium faciebat exterior. ${ }^{16}$

Trattandosi di due descrizioni topiche della bellezza femminile, è del tutto ovvio che queste presentino una serie di coincidenze. L'enumerazione delle parti anatomiche doveva, infatti, secondo quanto previsto dal canone, svolgersi a partire dalla testa. Ed è quanto puntualmente si verifica nei due testi presi in considerazione. Se, dunque, in questo caso è d'obbligo una certa prudenza nel proporre quale fonte della descriptio achilliniana quella che si legge al principio dell'Historia, è tuttavia innegabile che queste due rappresentazioni della bellezza si svolgono esibendo quasi sempre gli stessi elementi secondo un identico ordine:

$\begin{array}{ll}\text { AcHillini } & \text { Piccolomini } \\ \text { capelli } & \text { capelli } \\ \text { fronte } & \text { fronte } \\ \text { ciglia } & \text { ciglia } \\ \text { occhi } & \text { occhi } \\ \text { naso } & \text { naso } \\ \text { guance } & \text { guance } \\ \text { labbra } & \text { bocca } \\ \text { fiato } & - \\ \text { bocca } & \text { labbra } \\ \text { denti } & \text { denti } \\ \text { parole } & \text { parole } \\ \text { mento } & \text { mento } \\ \text { collo } & \text { collo } \\ \text { gola } & - \\ \text { seno } & - \\ \text { mano } & - \\ \text { parti nascoste } & \text { parti nascoste }\end{array}$

La versione del Braccesi non presenta, a differenza di quanto si è potuto constatare a proposito della prima lettera di Eurialo a Lucrezia, alcun ampliamento o sviluppo rispetto al testo del Piccolomini. Per quanto riguarda, 
quindi, il ternario Maraviglia non è, mia alma diva non è possibile stabilire se l'Achillini per la sua composizione abbia fatto ricorso anche al volgarizzamento-rifacimento del fiorentino.

In questi ultimi anni si è, a più riprese, sottolineata l'amplissima fortuna editoriale dell'Historia e della relativa versione procurata dal Braccesi: piace, proprio per questo, essere riusciti a produrre due testimonianze (sia pur di peso diverso) che questa fortuna esercito la sua influenza anche sulla contemporanea elegia in volgare.

Andrea Comboni 
I. Cfr. A. Comboni, Orfeo nell'inedito "Triumpho de crudelitate" di Giovanni Filoteo Acbillini, «Versants», 24 (1993), pp. 91-10s e Le elegie di Giovanni Filoteo Acbillini, in L'elegia nella tradizione poetica italiana, a cura di A. Comboni - A. Di Ricco, Trento, 2003, pp. 147-75.

2. In una lettera del 5 novembre 1504 , indirizzata a Isabella d'Este, il Calmeta indicava con chiarezza il legame tra il capitolo ternario e l'elegia: «Hanno li moderni poi e contemporanei nostri (o sia per la sonorità de la terza rima, o vero perché el terzetto più cum la musica abia conformitade) a li ternari l'officio de la elegia assignato» (Vincenzo Calmeta, Prose e lettere edite e inedite (con due appendici di altri inediti), a cura di C. Grayson, Bologna, 1959, p. 52).

3. Cfr. Comboni, Le elegie di Giovanni Filoteo Achillini cit., pp. I 56-75.

4. Sulla fortuna delle epistole ovidiane durante la seconda metà del Quattrocento cfr. lo studio di H. Dorrie, Der heroische Brief: Bestandsaufnabme, Geschicbte, Kritike einer bumanistischbarocken Literaturgattung, Berlin, 1968.

5. L'epistola, purtroppo mutila, si legge alle cc. 86 v-87 v del manoscritto Acquisti e Doni 397 della Biblioteca Medicea Laurenziana di Firenze. Nella trascrizione di questo, come dei successivi testi, mi sono limitato a separare e unire le parole ed a introdurre segni interpuntivi e diacritici secondo l'uso moderno; ho inoltre distinto $u$ da $v$ e ho trascritto $-i j$ con $-i i$.

6. Il sintagma «agnizione di lettura» deriva, ovviamente, dal bel saggio di G. Nencioni, Agnizioni di lettura, «Strumenti critici», 2 (febbraio 1967), pp. 191-98.

7. Come ha rilevato M. L. Doglio, «nel periodo fra il 1483 e il I 500 si successero oltre trenta stampe (per citare solo quelle di cui restano testimonianze) ed oltre quaranta fra il I 500 e il i6oo» (M. L. Doglio, Introdurione, in Enea Silvio Piccolomini, Storia di due amantie Rimedio d'amore, Traduzione e introduzione di M. L. Doglio, con un saggio di L. Firpo, Torino, 1973 , p. I 2); cfr. anche G. Albanese, Da Petrarca a Piccolomini: codificazione della novella umanistica, in Favole, parabole, istorie. Le forme della scrittura novellistica dal Medioevo al Rinascimento, Atti del Convegno di Pisa (26-28 ottobre 1998), a cura di G. Albanese, L. Battaglia Ricci e R. Bessi, Roma, 2000, pp. 292-95.

8. Doglio, Introdu₹ione cit., p. 5 .

9. Piccolomini, Storia di due amanti, p. 48. Si riporta la traduzione di M. L. Doglio: «Lucrezia mia, ti augurerei salute con le mie parole se mi restasse un po' di salute, ma la mia salute e ogni mia speranza di vivere dipendono da te. Io ti amo più di me stesso e credo che il mio amore non ti sia nascosto. Il mio viso, spesso rigato di lacrime, e i sospiri emessi in tua presenza possono testimoniare la ferita che ho nel cuore. Permetti, ti prego, che mi apra a te. La tua straordinaria bellezza mi ha rapito e mi tiene prigioniero con la tua grazia superiore al fascino d'ogni altra. Prima d'ora non sapevo cosa fosse l'amore, tu mi hai sottomesso al dominio della passione. Ho lottato a lungo, lo confesso, per sottrarmi al giogo crudele. Ma lo splendore del tuo sguardo ha vinto i miei sforzi con i tuoi occhi sfolgoranti più potenti dei raggi del sole. Sono tuo schiavo e non sono più padrone di me, tu mi hai tolto il sonno e l'appetito. Giorno e notte ti amo, ti desidero, ti invoco, ti aspetto, penso a te, spero in te, in te sta ogni mia gioia; il mio animo è tuo, ti appartengo interamente, tu sola puoi salvarmi o perdermi. Scegli una di queste alternative e fammi sapere la tua decisione. Non essere con le parole più crudele di quanto lo sei stata con gli occhi avvincendomi a te. Non ti chiedo una cosa impossibile, ti domando solo di poter parlare con te. Questo soltanto vuole la mia lettera: dirti a voce quello che ora ti scrivo. Se me lo concedi, mi fai vivere e mi rendi felice. Se me lo neghi, il mio cuore cessa di battere perché ama più te di me. Mi raccomando a te e alla tua lealtà. Sta bene, anima mia e sostegno della mia vita» (Piccolomini, Storia di due amanti, p. 49). 


\section{Piccolomini, Braccesi e Achillini}

I . Sul volgarizzamento-rifacimento del Braccesi cfr. M. P. Mussini Sacchi, Le rime "necessarie" nel romanzo quattrocentesco, in Il libro di poesia dal copista al tipografo, a cura di M. Santagata e A. Quondam, Modena-Ferrara, I989, pp. i i I- I6 e, soprattutto, N. Tonelli, L'Historia di due amanti di Alessandro Braccesi, in Favole, parabole, istorie cit., pp. 337-57; sulla fortuna di questo testo cfr. A. Perosa, Braccesi, Alessandro, in Dizionario biografico degli italiani, I 3, Roma, I 97 I, p. 607 e M. Masoero, Novella in versi e prosimetro: riscritture volgari dell'Historia de duobus amantibus del Piccolomini, in Favole, parabole, istorie cit., pp. 317-25.

I I. Per il volgarizzamento-rifacimento del Braccesi ho utilizzato la seguente edizione: Epistole e sonecti d'amore, Battista Farfengo, Brescia, 3 I-X-I 49I, c. as v.

I 2. Sul Viridario ha di recente richiamato l'attenzione degli studiosi Silvia Longhi per l'insolito dato strutturale che lo contraddistingue: «il tessuto delle ottave di questo poema include cinque capitoli ternari, più precisamente cinque epistole, scritte da personaggi della storia» (S. Longhi, Lettere a Ippolito e a Teseo, in Le memorie antiche. Modelli classici da Petrarca a Tassoni, Verona, 2001, pp. 49-50).

I3. Giovanni Filoteo Achillini, Viridario, per Hieronymo di Plato, Bologna, is i 3 , c. CXXXII r-v.

I 4. Alle cc. I 32 r-I 33 r, I 45 r-v (a causa di una scompaginazione dell'ordine originale delle carte). Al v. 35 si corregge la lezione del ms. «ad arte».

I 5. Sulla descriptio pulchritudinis nella poesia quattro-cinquecentesca cfr. G. Pozzi, Il ritratto della donna nella poesia d'inizio Cinquecento e la pittura di Giorgione e Nota additiva alla descriptio puellae, in Sull'orlo del visibile parlare, Milano, I993, pp. I45-7 I, I73-84; A. Quondam, Il naso di Laura. Considerazioni sul ritratto poetico e la comunicazione lirica, in Il naso di Laura. Lingua e poesia lirica nella tradizione del Classicismo, Modena-Ferrara, I 99I, pp. 291-328.

I6. Piccolomini, Storia di due amanti, p. 30. Si riporta la traduzione di M. L. Doglio: «Era di statura più alta delle altre, aveva capelli lunghi e folti, simili a lamine d'oro che portava sciolti all'indietro, non al modo delle vergini, ma annodati con pietre preziose, fronte spaziosa e ben proporzionata, senza alcuna ruga, sopracciglia arcuate, scure e sottili, separate a giusta distanza, occhi così luminosi e sfolgoranti che abbagliavano come il sole chi li guardava. Solo con lo sguardo essa poteva uccidere a suo piacere e a suo piacere richiamare i morti alla vita. Il naso era dritto, gli zigomi rosei, perfetti. Nulla pareva più amabile né più piacevole a vedersi delle deliziose fossette che vi si formavano quando sorrideva. Nessuno poteva vederle senza desiderare di baciarle. La bocca era piccola, leggiadra, le labbra di corallo fatte per i morsi, i denti piccoli, regolari, sembravano di cristallo e la lingua, muovendosi lievemente tra di essi, mandava fuori non parole ma dolcissime armonie. Che dire della forma del mento e del candore del collo? Ogni parte del suo corpo richiederebbe una lode. Quello che si vedeva all'esterno lasciava indovinare le bellezze nascoste» (PiccoLOMINI, Storia di due amanti, p. 3I). 